\title{
Complex scalar field in strictly stationary Einstein-Maxwell-axion-dilaton spacetime with negative cosmological constant
}

\author{
Bartlomiej Bakon and Marek Rogatko \\ Institute of Physics \\ Maria Curie-Sklodowska University \\ 20-031 Lublin, pl. Marii Curie-Sklodowskiej 1, Poland \\ marek.rogatko@poczta.umcs.lublin.pl \\ rogat@kft.umcs.lublin.pl \\ (Dated: November 15, 2018)
}

\begin{abstract}
We proved that strictly stationary Einstein-Maxwell-axion-dilaton spacetime with negative cosmological constant could not support a nontrivial configurations of complex scalar fields. We considered the general case of the arbitrary number of $U(1)$-gauge fields in the theory under consideration.

PACS numbers: 04.20.-q, 04.20.Cv, 04.50.Gh
\end{abstract}

\section{INTRODUCTION}

One of the most important issues of general relativity or its generalization to higher dimensions are connected with a gravitational collapse and the emergence of black holes or black objects. Recently, self-gravitating objects and gravitational collapse in asymptotically anti-de Sitter (AdS) spacetime gain much attention. One of the reason is that there is a surprising connection between general relativity and non-gravitational fields of physics. An exact correspondence between gravity theory in $(d+1)$-dimensional AdS spacetime and a conformal field theory of the boundary of this spacetime was proposed [1]. The AdS/CFT correspondence becomes a very useful tool which helps us to understand the strong coupled gauge theories as well as it has been applied to the condensed matter physics, including superconductivity and superfluidity.

In Ref. 2] it was shown that the only strictly stationary asymptotically AdS spacetime fulfilling the vacuum Einstein equations with negative cosmological constant is the AdS spacetime. Uniqueness of AdS spacetime in higher dimensions was studied in [3], where it was also revealed that the vacuum solution of Einstein equations with a negative cosmological constant is AdS spacetime. The global aspects of the complete nonsingular asymptotically locally AdS spacetime being the solution of Einstein vacuum equations were elaborated in Ref. [4]. On the other hand, the properties and uniqueness of AdS solitons were considered in [5], while the problem of non-existence of black holes under certain asymptotic conditions was tackled in [6]. The systematic classification of static vacuum spacetimes with negative cosmological constant was conducted in Ref. [7].

A different issue, related to the problem of gravitational collapse in generalization of Einstein theory to higher dimensions and emergence of higher dimensional black objects (like black rings, black Saturns) and multidimensional black holes was widely studied. The complete classification of $n$-dimensional charged black holes both with nondegenerate and degenerate component of the event horizon was proposed in Refs. [8], while partial results for the very nontrivial case of $n$-dimensional rotating black hole uniqueness theorem were provided in [9]. These researches comprise also the case of the low-energy limit of the string theory, like dilaton gravity, Einstein-Maxwell-axiondilaton (EMAD)-gravity and supergravities theories [10]. On the other hand, black holes and their properties as key ingredients of the AdS/CFT attitude to superconductivity also acquire great attention. The nonlinear evolution of a weakly perturbed AdS spacetime by solving numerically spherically symmetric Einstein massless scalar field was studied in [11]. It was shown that AdS spacetime is unstable under arbitrary small generic perturbations, both in four and higher dimensions [12. Recently, the numerical solutions of black holes with only one Killing vector were presented [13]. This investigation was motivated by the superradiance arguments 14 that AdS black hole might exist having only one Killing vector. Moreover, topological defects like vortex [15] or interaction domain walls with AdS black holes [16] were also taken into account. These works naturally pose a question about possible configurations in AdS spacetime with certain matter fields. In Ref. 17] it was revealed that strictly stationary AdS spacetime could not allow for the existence of nontrivial configurations of complex scalar fields or form fields.

Motivated by the aforementioned problems of strictly stationary AdS spacetimes with matter as well as the wide range of possible applications of the AdS/CFT correspondence, we shall investigate the problem of possible matter configurations in EMAD-gravity. EMAD-gravity is the low-energy limit of heterotic string compactified to fourdimensions. For the generality of the researches we shall not restrict our consideration to only one gauge field. One takes into account the arbitrary number of $U(1)$-gauge fields. In some sense we generalize considerations conducted on Eistein-Maxwell (EM) background [17] to the more complicated gravity theory.

Our paper is organized as follows. In Sec.II we describe theory under consideration in terms of $U(1)$-gauge field 
strengths and their $S L(2, R)$-duals. Then, using the rigid positive energy theorem [18], we find that strictly stationary EMAD spacetime with negative cosmological constant cannot be nontrivial configurations of complex scalar field. Then, one concludes the investigations.

\section{EMAD-GRAVITY}

Motivated by the recent works concerning the numerical solutions of Einstein complex scalar field systems we shall consider the generalization of Einstein-Maxwell (EM) complex scalar field system [13] to the low-energy limit of the heterotic string theory, the so-called Einstein-Maxwell-axion-dilaton gravity (EMAD) with cosmological constant. Moreover, we take into account complex scalar field existing on this background. The theory under consideration will contain gravitation field $g_{\mu \nu}$, arbitrary number $N$ of $U(1)$-gauge fields, the dilaton field $\phi$ and axion $a$. The action will be provided by [19]

$$
\begin{aligned}
S=\int d^{4} x \sqrt{-g}[R & -2 \Lambda-2 \nabla_{\mu} \phi \nabla^{\mu} \phi-\frac{1}{2} \nabla_{\mu} a \nabla^{\mu} a-\sum_{n=1}^{N} e^{-2 \phi} F_{\mu \nu}^{(n)} F^{\mu \nu(n)}-\sum_{n=1}^{N} a F_{\mu \nu}^{(n)} * F^{\mu \nu}(n) \\
& \left.-2|\nabla \pi|^{2}\right]
\end{aligned}
$$

where we have denoted the strength of the adequate gauge field $F_{\mu \nu}^{(n)}=2 \nabla_{[\mu} A_{\nu]}^{(n)}$. Its dual is given by * $F_{\mu \nu}^{(n)}=$ $\frac{1}{2} \epsilon_{\mu \nu \alpha \beta} F^{\mu \nu(n)}$ and by $\pi$ we denote a complex scalar field. One should remark that when the number of vector fields is six we obtain $N=4, d=4$ bosonic part of supergravity theory. For the sake of generality one will keep the number of $U(1)$-gauge fields $n$ to be arbitrary.

In many physical problems [19] the action describing by the relation (1) can be written in a more convenient form. Namely, introducing a complex scalar axi-dilaton which implies

$$
\lambda=a+i e^{-2 \phi}
$$

and defining $S L(2, R)$-duals to the gauge fields $F_{\mu \nu}^{(n)}$, the action in question yields

$$
S=\int d^{4} x \sqrt{-g}\left[R-2 \Lambda+2 \frac{\nabla_{\mu} \lambda \nabla^{\mu} \tilde{\lambda}}{(\lambda-\tilde{\lambda})^{2}}+\sum_{n=1}^{N} F_{\mu \nu}^{(n)} * \tilde{\mathcal{F}}^{\mu \nu(n)}-|\nabla \pi|^{2}\right],
$$

where the $S L(2, R)$-duals are provided by the relation

$$
\tilde{\mathcal{F}}_{\alpha \beta}^{(n)}=e^{-2 \phi} * F_{\alpha \beta}^{(n)}-a F_{\alpha \beta}^{(n)}
$$

The equation of motion for $S L(2, R)$-duals is of the form $\nabla_{\alpha} * \tilde{\mathcal{F}}^{\alpha \beta(n)}=0$ which entails the existence of $N$ vector potentials $\tilde{\mathcal{A}}_{\beta}^{(n)}$ satisfying relation of the form

$$
\tilde{\mathcal{F}}_{\alpha \beta}^{(n)}=2 \nabla_{[\alpha} \tilde{\mathcal{A}}_{\beta]}^{(n)}
$$

Consequently, the analogous relation for $F_{\mu \nu}^{(n)}=2 \nabla_{[\mu} A_{\nu]}^{(n)}$ is not a consequence of equations of motion but it stems from the Bianchi identity.

The energy-momentum tensor components in the theory in question $T_{\alpha \beta}(F, \tilde{\mathcal{F}}, \lambda, \Pi)=T_{\alpha \beta}(\Pi)+T_{\alpha \beta}(F, \tilde{\mathcal{F}}, \lambda)$ are given respectively

$$
T_{\alpha \beta}(\pi)=-2 g_{\alpha \beta} \nabla_{\gamma} \pi \nabla^{\gamma} \pi^{*}+2\left(\nabla_{\alpha} \pi \nabla_{\beta} \pi^{*}+\nabla_{\alpha} \pi^{*} \nabla_{\beta} \pi\right),
$$

for the complex scalar field, while for the $U(1)$-gauge fields may be written in the form as follows:

$$
T_{\alpha \beta}(F, \tilde{F}, \lambda)=-\left[4 \sum_{n=1}^{N} F_{\alpha \delta(n)} * \tilde{\mathcal{F}}_{\beta}{ }^{(n)}-g_{\alpha \beta} \sum_{n=1}^{N} F_{\alpha \beta}^{(n)} * \tilde{\mathcal{F}}^{\alpha \beta(n)}\right]+\frac{2 g_{\alpha \beta} \nabla_{\gamma} \lambda \nabla^{\gamma} \bar{\lambda}-4 \nabla_{\alpha} \lambda \nabla_{\beta} \bar{\lambda}}{(\lambda-\bar{\lambda})^{2}}
$$

In our paper we shall examine the strictly stationary spacetime, which is not restricted to the axisymmetric ones and does not contain any black hole. The strictly stationary spacetime by the definition posses everywhere a timelike 
Killing vector field $k_{\delta}$. Due to the strictly stationarity, one assumes that all the field in the considered theory will be stationary. Namely, one has that

$$
\mathcal{L}_{k} F_{\alpha \beta}=\mathcal{L}_{k} \tilde{\mathcal{F}}_{\alpha \beta}=\mathcal{L}_{k} a=\mathcal{L}_{k} \phi=\mathcal{L}_{k} \Pi=0
$$

It can be proved, having in mind the exact form of the energy-momentum tensor $T_{\alpha \beta}(F, \tilde{\mathcal{F}}, \lambda, \Pi)$ that it will satisfy the stationarity assumption, i.e., $\mathcal{L}_{k} T_{\alpha \beta}(F, \tilde{\mathcal{F}}, \lambda, \Pi)=0$.

Furthermore one can define the twist vector $\omega_{a}$ of stationary Killing vector field $k_{a}$ by the following definition:

$$
\omega_{a}=\frac{1}{2} \epsilon_{a b c d} k^{b} \nabla^{c} k^{d} .
$$

Using the formula valid for any Killing vector field $\nabla_{\alpha} \nabla_{\beta} \chi_{\gamma}=-R_{\beta \gamma \alpha}{ }^{\delta} \chi_{\delta}$, and carrying out the differentiation of the twist vector, one leads to the identity satisfied by the stationary Killing vector field $k_{\alpha}$

$$
\nabla_{\beta} \omega_{\alpha}=\frac{1}{2} \epsilon_{\alpha \beta \gamma \delta} k^{\gamma} R^{\delta \chi} k_{\chi}
$$

By virtue of the definition of $\omega_{\alpha}$ it follows directly that the following yields:

$$
\nabla_{\alpha}\left(\frac{\omega^{\alpha}}{V^{4}}\right)=0
$$

where one denotes $V^{2}=-k_{\gamma} k^{\gamma}$.

To proceed further, we define electric and magnetic components for gauge field strengths $F_{\alpha \beta}^{(n)}$ and $\tilde{\mathcal{F}}_{\alpha \beta}^{(n)}$. Namely, for electric components we have

$$
E_{\alpha}^{(n)}=-F_{\alpha \beta}^{(n)} k^{\beta}, \quad \tilde{E}_{\alpha}^{(n)}=-\tilde{\mathcal{F}}_{\alpha \beta}^{(n)} k^{\beta}
$$

while magnetic ones are provided by

$$
B_{\alpha}^{(n)}=\frac{1}{2} \epsilon_{\alpha \beta \gamma \delta} k^{\beta} F^{\gamma \delta(n)}, \quad \tilde{B}_{\alpha}^{(n)}=\frac{1}{2} \epsilon_{\alpha \beta \gamma \delta} k^{\beta} \tilde{\mathcal{F}}^{\gamma \delta(n)} .
$$

Consequently, in terms of the above magnetic and electric components, the field strength $F_{\alpha \delta}^{(n)}$ is given by the following relation:

$$
V^{2} F_{\alpha \beta}^{(n)}=-2 k_{[\alpha} E_{\beta]}^{(n)}+\epsilon_{\alpha \beta \gamma \delta} k^{\gamma} B^{\delta(n)}
$$

while for $S L(2, R)$-duals, one obtains that $\tilde{\mathcal{F}}_{\alpha \delta}^{(n)}$ yields

$$
V^{2} \tilde{\mathcal{F}}_{\alpha \beta}^{(n)}=-2 k_{[\alpha} \tilde{E}_{\beta]}^{(n)}+\epsilon_{\alpha \beta \gamma \delta} k^{\gamma} \tilde{B}^{\delta(n)} .
$$

On the other hand, equations of motion for magnetic and electric parts for each gauge field strength imply

$$
\begin{aligned}
& \nabla_{\alpha}\left(\frac{\tilde{E}^{\alpha(n)}}{V^{2}}\right)=2 \frac{\tilde{B}^{\gamma(n)}}{V^{2}} \omega_{\gamma}, \\
& \nabla_{\alpha}\left(\frac{E^{\alpha(n)}}{V^{2}}\right)=2 \frac{B^{\gamma(n)}}{V^{2}} \omega_{\gamma}, \\
& \nabla_{\alpha}\left(\frac{\tilde{B}^{\alpha(n)}}{V^{2}}\right)=-2 \frac{\tilde{E}^{\gamma}(n)}{V^{2}} \omega_{\gamma}, \\
& \nabla_{\alpha}\left(\frac{B^{\alpha(n)}}{V^{2}}\right)=-2 \frac{E^{\gamma}(n)}{V^{2}} \omega_{\gamma} .
\end{aligned}
$$

By virtue of the field invariance conditions $\mathcal{L}_{k} F_{\alpha \beta}^{(n)}=\mathcal{L}_{k} \tilde{\mathcal{F}}_{\alpha \beta}^{(n)}=0$, as well as relations $\nabla_{[\gamma} F_{\alpha \beta]}^{(n)}=\nabla_{[\gamma} \tilde{\mathcal{F}}_{\alpha \beta]}^{(n)}=0$, one achieves the generalized Maxwell source-free equations. Their explicit forms are provided by

$$
\begin{aligned}
& \nabla_{[\alpha} E_{\beta]}^{(n)}=\nabla_{[\alpha} \tilde{E}_{\beta]}^{(n)}=0, \\
& \nabla_{[\alpha} B_{\beta]}^{(n)}=\nabla_{[\alpha} \tilde{B}_{\beta]}^{(n)}=0 .
\end{aligned}
$$


The demand that the spacetime under consideration is simply connected, enables one to introduce the adequate potential for each electric and magnetic components. They are obliged to satisfy

$$
\begin{array}{ll}
E_{\alpha}^{(n)}=\nabla_{\alpha} \varphi^{(n)}, & \tilde{E}_{\alpha}^{(n)}=\nabla_{\alpha} \Phi^{(n)}, \\
B_{\alpha}^{(n)}=\nabla_{\alpha} \psi^{(n)}, & \tilde{B}_{\alpha}^{(n)}=\nabla_{\alpha} \Psi^{(n)} .
\end{array}
$$

Taking into account Eq. (10) and the explicit form of the Ricci tensor for the fields in question, we can assert that the following equality takes place:

$$
\nabla_{[\alpha} \omega_{\beta]}=4 \sum_{n=1}^{N} B_{[\alpha}^{(n)} \tilde{B}_{\beta]}^{(n)}
$$

The right-hand side of (24) depicts the generalized Poynting flux in EMAD-gravity.

The fact that electric and magnetic part of the adequate gauge field strength can be expressed in terms of the potentials, enables one to find other relations binding them with the aforementioned twist vector. Thus, by the direct calculations it can be revealed that one arrives at the relations binding together the twist vector, the potentials $\psi^{(n)}$ and $\tilde{B}_{\beta}^{(n)}$

$$
\nabla_{[\alpha}\left(\omega_{\beta]}+2 \sum_{n=1}^{N} \psi^{(n)} \tilde{B}_{\beta]}^{(n)}\right)=0
$$

and the other for $\Psi^{(n)}$ and $B_{\beta}^{(n)}$

$$
\nabla_{[\alpha}\left(\omega_{\beta]}-2 \sum_{n=1}^{N} \Psi^{(n)} B_{\beta]}^{(n)}\right)=0
$$

Consequently, the existence of the following scalar functions are guaranteed

$$
\begin{aligned}
& \nabla_{\alpha} U_{B^{(n)}}=\omega_{\alpha}+2 \sum_{n=1}^{N} \psi^{(n)} \tilde{B}_{\alpha}^{(n)}, \\
& \nabla_{\alpha} U_{\tilde{B}^{(n)}}=\omega_{\alpha}-2 \sum_{n=1}^{N} \Psi^{(n)} B_{\alpha}^{(n)} .
\end{aligned}
$$

Having in mind the generalized forms of Maxwell equations in the theory in question, one is able to find the following relations fulfilling by $U_{B^{(n)}}$

$$
\nabla_{\alpha}\left(U_{B^{(n)}} \frac{\omega^{\alpha}}{V^{4}}-\sum_{n=1}^{N} \frac{\psi^{(n)} \tilde{E}^{\alpha(n)}}{V^{2}}\right)=\frac{\omega_{\beta} \omega^{\beta}}{V^{4}}-\sum_{n=1}^{N} \frac{B_{\alpha}^{(n)} \tilde{E}^{\alpha(n)}}{V^{2}},
$$

and by the scalar function $U_{\tilde{B}^{(n)}}$

$$
\nabla_{\alpha}\left(U_{\tilde{B}^{(n)}} \frac{\omega^{\alpha}}{V^{4}}+\sum_{n=1}^{N} \frac{\Psi^{(n)} E^{\alpha(n)}}{V^{2}}\right)=\frac{\omega_{\beta} \omega^{\beta}}{V^{4}}+\sum_{n=1}^{N} \frac{\tilde{B}_{\alpha}^{(n)} E^{\alpha(n)}}{V^{2}} .
$$

Further, combining the above expressions in order to find $2 R_{\alpha \beta} k^{\alpha} k^{\beta} / V^{2}$, one obtains the relation of the form as

$$
\nabla_{\alpha}\left(\frac{\nabla^{\alpha} V^{2}}{V^{2}}+4 \frac{\omega_{\alpha} \omega^{\alpha}}{V^{4}}\right)=-2 \Lambda+2 \sum_{n=1}^{N} \frac{\tilde{E}_{\beta}^{(n)} B^{\beta(n)}-E_{\beta}^{(n)} \tilde{B}^{\beta(n)}}{V^{2}} .
$$

Combining Eqs.(29)-(30) and (31), by a direct calculation, we reach to the following expression valid for each of the gauge fields in question:

$$
\nabla_{\alpha}\left(\frac{\nabla^{\alpha} V^{2}}{V^{2}}+\Theta^{\alpha(n)}\right)=-2 \Lambda
$$


where we have denoted by $\Theta^{\alpha(n)}$

$$
\Theta^{\alpha(n)}=2\left(U_{B^{(n)}}+U_{\tilde{B}^{(n)}}\right) \frac{\omega^{\alpha}}{V^{4}}+2 \sum_{n=1}^{N} \frac{\left(\Psi^{(n)} E^{\alpha(n)}-\psi^{(n)} \tilde{E}^{\alpha(n)}\right)}{V^{4}} .
$$

Considering the case when $\Lambda<0$, one can follow Ref. [17] and introduce the vector field $r^{\beta}$, provided by

$$
\nabla_{\gamma} r^{\gamma}=-2 \Lambda
$$

As in Ref. 2] one can define a spacetime which is AdS if there exists a chart $(t, r, \theta, \phi)$ defined outside a spatial compact world tube. The line element of it is subject to the relation

$$
\begin{aligned}
d s^{2}=d s_{0}^{2} & +\mathcal{O}\left(r^{-2}\right) d t^{2}+\mathcal{O}\left(r^{-1}\right) d r^{2} \\
& +\mathcal{O}(r)(\text { remaining differentials not including } d r) \\
& +\mathcal{O}\left(r^{-1}\right)(\text { remaining differentials not including } d r)
\end{aligned}
$$

where $d s_{0}^{2}$ is Schwarzschild-anti-de Sitter metric. On the other hand, near infinity $r^{\alpha}$ will imply

$$
r^{\alpha} \simeq \Lambda r\left(\frac{\partial}{r}\right)^{\alpha}+\ldots
$$

The global existence of $r^{\alpha}$ is justified by the assumption that $r^{\alpha}=\nabla^{\alpha} \chi$, where on its turn $\chi$ fulfils the equation $\nabla^{2} \chi=-2 \Lambda$. Just, one can redefine $\chi$ in order to subtracted the term $-2 \Lambda$ and obtain $\nabla^{2} \tilde{\chi}=S$, where $S$ is a nonsingular source term. The existence of the solution of the above equation is guaranteed on a regular Riemaniann manifold, so one can always introduce $r^{\alpha}$.

It implies that Eq.(32) can be reorganize to the following form:

$$
\nabla_{\alpha}\left(\frac{\nabla^{\alpha} V^{2}}{V^{2}}-r^{\alpha}+\Theta^{\alpha(n)}\right)=0
$$

The value of the left-hand side of the above integral can be rewritten in the form of the surface integral proportional to the total mass $M$.

Having in mind the rigid positive energy theorem [18], and taking into account the volume integral of relation (37) which it equal to zero, one concludes that $M=0$. It yields that the spacetime under consideration should be the exact anti-de-Sitter one. One remarks that we get no nontrivial self-gravitating solutions for complex scalar fields in EMAD-gravity with negative cosmological constant, under the assumptions imposed here, i.e., under the strictly stationarity of the considered spacetime.

Summing up, we have revealed that the strictly stationary, simply connected spacetime in EMAD-gravity with positive cosmological constant have no nontrivial configurations of complex scalar fields. The spacetime under consideration ought to reduce to be exactly Minkowski or anti-de Sitter depending on the occurrence of negative cosmological constant.

\section{CONCLUSIONS}

In our paper we have considered the problem of the possible emergence of a self-gravitating structure composed of complex scalar fields. We considered EMAD-gravity being the low-energy limit of heterotic string theory, which constitutes the generalization of EM theory with additional fields of axion and dilaton. One takes into account the case of negative cosmological constant. For the sake of generality of our investigations, we elaborated the case of an arbitrary number of $U(1)$-gauge fields. One restricts his consideration to the case when the spacetime in question is strictly stationary, i.e., if it admits nowhere vanishing timelike Killing vector field. Rewriting the action describing the considered physical system in terms of $U(1)$-gauge strengths and their $S L(2, R)$-duals, enables us to implement the rigid positive energy theorem [18] and conclude that strictly stationary EMAD spacetime with negative cosmological constant could not support nontrivial configurations of complex scalar fields. It means that, as well as in the case of EM theory with negative cosmological constant, one comes to a conclusion that a self-gravitating complex scalar fields cannot exist in the theory in question.

Of course, it remains for the future investigations how to overcome the problem of getting rid of the assumption (34). We hope to return to this problem elsewhere. 


\section{Acknowledgments}

MR was partially supported by the grant of the National Science Center 2011/01/B/ST2/00408.

[1] J.M.Maldacena, Adv. Theor. Math. Phys. 2, 231 (1998), S.s.Gubser, I.R.Klebanov, and A.M.Polyakov, Phys. Lett. B 428, 105 (1998), S.A.Hartnoll, Class. Quantum Grav. 26, 224002 (2009), G.T.Horowitz, Class. Quantum Grav. 28, 114008 (2011), G.T.Horowitz, Introduction to Holographic Superconductors, hep-th $\mathbf{1 0 0 2 . 1 7 2 2}$ (2010).

[2] W.Boucher, G.W.Gibbons, and G.T.Horowitz, Phys. Rev. D 30, 2447 (1984).

[3] J.Qing, Ann. Henri Poincare 5, 245 (2004), X.D.Wang, Acta Math. Sin. 21, 917 (2005).

[4] M.T.Anderson, Class. Quantum Grav. 23, 6935 (2006).

[5] G.J.Galloway, S.Surya, and E.Woolgar, Phys. Rev. Lett. 88, 101102 (2002), G.J.Galloway, S.Surya, and E.Woolgar, Commun. Math. Phys. 241, 1 (2003).

[6] G.J.Galloway, S.Surya, and E.Woolgar, Class. Quantum Grav. 20, 1635 (2003).

[7] P.T.Chruściel and W.Simon, J. Math. Phys. 42, 1779 (2001).

[8] G.W.Gibbons, D.Ida, and T.Shiromizu, Phys. Rev. D 66, 044010 (2002), G.W.Gibbons, D.Ida, and T.Shiromizu, Phys. Rev. Lett. 89, 041101 (2002), S.Hollands, A.Ishibashi, and R.M.Wald, Commun. Math. Phys. 271, 699 (2007), M.Rogatko, Class. Quantum Grav. 19, L151 (2002), M.Rogatko, Phys. Rev. D 67, 084025 (2003), M.Rogatko, ibid. 70, 084025 (2004), M.Rogatko, ibid. 73, 124027 (2006).

[9] Y.Morisawa and D.Ida, Phys. Rev. D 69, 124005 (2004), Y.Morisawa, S.Tomizawa, and Y.Yasui, Phys. Rev. D 77, 064019 (2008), M.Rogatko, Phys. Rev. D 77, 124037 (2008), S.Hollands and S.Yazadjiev, Commun. Math. Phys. 283, 749 (2008), S.Hollands and S.Yazadjiev, Class. Quantum Grav. 25, 095010 (2008), D.Ida, A.Ishibashi, and T.Shiromizu, Prog. Theor. Phys. Suppl. 189, 52 (2011).

[10] A.K.M.Massod-ul-Alam, Class. Quantum Grav. 14, 2649 (1993), M.Mars and W.Simon, Adv. Theor. Math. Phys. 6, 279 (2003), M.Rogatko, Class. Quantum Grav. 14, 2425 (1997), M.Rogatko, Phys. Rev. D 58, 044011 (1998), M.Rogatko, ibid. 59, 104010 (1999), M.Rogatko, ibid. 82, 044017 (2010), M.Rogatko, Class. Quantum Grav. 19, 875 (2002), S.Tomizawa, Y.Yasui, and A.Ishibashi, Phys. Rev. D 79, 124023 (2009), S.Tomizawa, Y.Yasui, and A.Ishibashi, Phys. Rev. D 81, 084037 (2010), J.B.Gutowski, JHEP 0408, 049 (2004), J.P.Gauntlett, J.B.Gutowski, C.M.Hull, S.Pakis, and H.S.Real, Class. Quantum Grav. 20, 4587 (2003).

[11] P.Bizoń and A.Rostworowski, Phys. Rev. Lett. 107, 031102 (2011).

[12] J.Jalmużna, A.Rostworowski, and P.Bizoń, Phys. Rev. D 84, 085021 (2011).

[13] O.J.C.Dias, G.T.Horowitz, and J.E.Santos, JHEP 07, 115 (2011).

[14] H.K.Kunduri, J.Lucietti, and H.S.Reall, Phys. Rev. D 74, 084021 (2006).

[15] M.H.Dehghani, A.M.Ghezelbash, and R.B.Mann, Nucl. Phys. B 625, 389 (2002).

[16] R.Moderski and M.Rogatko, Phys. Rev. D 74, 044002 (2006).

[17] T.Shiromizu, S.Ohashi, and R.Suzuki, Phys. Rev. D 86, 064041 (2012).

[18] R.Bartnik, Commun. Pure Appl. Math. 39, 661 (1986),

R.Schoen and S-T.Yau, Commun. Math. Phys. 65, 45 (1979),

E.Witten, Commun. Math. Phys. 80, 381 (1981).

[19] E.Bergshoeff, R.Kallosh, and T.Ortin, Nucl. Phys. B 478, 156 (1996), R.Kallosh, A.Linde, T.Ortin, A.Peet, and A.Van Proeyen, Phys. Rev. D 46, 5278 (1992), R.Kallosh and T.Ortin, ibid. 48, 742 (1993), T.Ortin, ibid. 47, 3136 (1993), R.Kallosh, D.Kastor, T.Ortin, and T.Torma, ibid. 50, 6374 (1994). 